九州大学学術情報リポジトリ

Kyushu University Institutional Repository

\title{
ON A CONVERGENCE OF MINIMIZING SEQUENCES FOR UNIFORMLY CONVEX FUNCTIONS
}

Tagawa, Shojiro

International Institute for Advanced Study of Social Information Science

https://doi.org/10.5109/13100

出版情報 : 統計数理研究. 17 (1/2)，pp. 1-16，1976-03. Research Association of Statistical Sciences

バージョン :

権利関係 : 


\title{
ON A CONVERGENCE OF MINIMIZING SEQUENCES FOR UNIFORMLY CONVEX FUNCTIONS
}

\author{
By
}

Shôjirô TAGAWA*

(Received December 22, 1974)

\section{§1. Introduction.}

In this paper, we shall introduce the notion of a uniformly convex function defined on a normed linear space, which is different from the one introduced by Levitin and Poljak [2]. We shall show that the uniform convexity is a sufficient condition for a convergence of minimizing sequences and for the existence of a unique minimum on a closed convex set in a real Banach space under some additional assumptions.

In Section 2, we shall introduce a uniformly quasi-convex function which is different from that presented by Poljak [3] and investigate the difference between the uniform convexity in our sense and strict quasi-convexity. In Section 3, we shall give some properties of convex and uniformly convex functions. We shall show that every minimizing sequence converges to a unique minimum for a uniformly convex function on a closed convex set in a Banach space under some additional assumptions in Section 4. It is also described that the projection theorem in a Hilbert space falls under the category of nonlinear programming introduced in [5] with the aid of the above results in Section 5.

We can immediately apply this argument to linear optimal control problems with uniformly convex cost criteria (for examples, $L_{p}$ norm ( $p \geqq 2$ ), integral quadratic form and so on) and to some problems of best approximation theory. This argument makes us possible to treat many optimization problems by a unified approach.

\section{§ 2. Uniformly quasi-convex functions.}

In this section, we shall introduce a new notion of uniformly quasi-convex functions and compare them with the other classes of functions.

Let $X$ be a normed linear space over a real number field $R$ and let $f$ be a realvalued function defined on $X$.

Definition 2.1. A real-valued function $f$ on $X$ is called to be uniformly quasiconvex if for every real number $d$ and every positive number $\varepsilon$, there exists a positive number $\delta=\delta(d, \varepsilon)$ such that

$$
f\left(\frac{x+y}{2}\right) \leqq d-\delta \quad \text { whenever } \quad f(x) \leqq d, f(y) \leqq d,\|x-y\| \geqq \varepsilon, x, y \in X .
$$

* International Institute for Advanced Study of Social Information Science, Fujitsu Limited, 17-25, Shinkamata 1-Chome, Ohta-ku, Tokyo, Japan. 
We shall present some classes of functions in order to clear the relations among them and our uniformly quasi-convex functions.

A function $f(x)$ is called to be uniformly quasi-convex in the sense of Poljak if $f\left(\frac{x+y}{2}\right) \leqq \max \{f(x), f(y)\}-\mu(\|x-y\|)$, where $\mu(\tau)$ is a real function, $\mu(0)=0$ and $\mu(\tau)>0$ for $\tau>0$. Then, it is evident that every uniformly quasi-convex function in the sense of Poljak is a uniformly quasi-convex function in our sense. But the converse is not true: For consider a real function $f(x)=e^{x}$. This function is uniformly quasi-convex in our sense, but not in the sense of Poljak.

A functional $f(x)$ is called to be strictly quasi-convex if

$$
f\left(\frac{x+y}{2}\right)<\max \{f(x), f(y)\} \quad \text { for all } \quad x, y(\neq x) \in X .
$$

It is then clear that every uniformly quasi-convex function is strictly quasi-convex. But the converse is not true: For consider the function $f: R^{2} \rightarrow R^{1}$ defined by

$$
f(z)=f(x, y)=e^{x^{2}+y} \quad \text { for all } z=(x, y) \in R^{2} .
$$

It is clear that this function is strictly quasi-convex. We shall show that this function is not uniformly quasi-convex in our sense. Let $d$ and $\varepsilon$ be arbitrary positive numbers and $w=(u, v)$ and $z=(x, y)$ two points in $R^{2}$ satisfying

$$
e^{x^{2}+y}=d, \quad e^{u^{2}+v}=d
$$

or

$$
y=-x^{2}+\log d, \quad v=-u^{2}+\log d,
$$

moreover

$$
\|w-z\|=\sqrt{(x-u)^{2}+(y-v)^{2}}=\varepsilon .
$$

Then we have

$$
(x-u)^{2}=\frac{\varepsilon^{2}}{1+(x+u)^{2}}
$$

and hence

$$
(x-u)^{2} \longrightarrow 0 \quad \text { as } \quad x, u \rightarrow \infty
$$

It is valid that

$$
f\left(\frac{w+z}{2}\right)=d \cdot e^{-\left(\frac{x-u}{2}\right)^{2}}
$$

so that

$$
f\left(\frac{w+z}{2}\right) \rightarrow d \quad \text { as } \quad x, u \rightarrow \infty,
$$

where $f(w)=f(z)=d$, and $\|w-z\|=\varepsilon$. This fact implies that this function $f(z)$ is not uniformly quasi-convex in our sense.

\section{§ 3. Uniformly convex functions.}

In this section we shall introduce the notion of uniformly convex functions and investigate the properties of these functions. 
Definition 3.1. Let $X$ be a normed linear space. The real-valued function $f$ defined over $X$ is called to be uniformly convex if $f$ is uniformly quasi-convex in the sense of Definition 2.1 and if $f$ is convex.

First of all, we shall present some properties of continuous convex functions defined over a normed linear space $X$. Throughout this section, we shall denote a real-valued convex function defined over a normed linear space $X$ by $f$ unless it is specifically mentioned. We shall presume the following assumption.

Assumption 1. There exists a real number $r_{0}$ such that the set $G_{r_{0}} \equiv\{x \in X \mid f(x)$ $\left.<r_{0}\right\}$ is a non-empty, bounded open set in $X$.

We now state the properties of a convex function under Assumption 1.

LEMMA 3.1. Under Assumption 1, $f$ is continuous on $X$.

PROOF. This is an immediate consequence of Proposition 21 in Bourbaki $[1 ; \mathrm{Ch}$. $\left.2, \S 2, n^{\circ} 10\right]$.

Q.E.D.

LEMMA 3.2. Under Assumption 1, choose a vector $x_{0} \in X$ such that $f\left(x_{0}\right)<r_{0}$. Then, for every vector $x \in X$ which is not the origin, there exists a positive number $\lambda_{x}$ satisfying $f\left(x_{0}+\lambda_{x} x\right)=r_{0}$. Moreover, $\lambda_{x}$ is unique to each $x \in X$.

Proof. Since the set $G_{r_{0}}$ is a bounded set in $X$, there is a positive number $b_{0}$ such that $\|x\| \leqq b_{0}$ for all $x \in G_{r_{0}}$. Hence, we have

$$
\|x\|>b_{0} \quad \text { implies } \quad x \notin G_{r_{0}}\left(\text { or } f(x) \geqq r_{0}\right) .
$$

Now, it is valid that to each vector $x(\neq 0) \in X$,

$$
\left\|x_{0}+\lambda x\right\|>b_{0} \quad \text { for sufficiently large } \lambda>0 \text {, }
$$

and, therefore,

$$
f\left(x_{0}+\lambda x\right) \geqq r_{0} .
$$

Since $f$ is continuous on $X$ and since $f\left(x_{0}\right)<r_{0}$, by Lemma 3.1, it is true that there is a small number $\lambda>0$ satisfying $f\left(x_{0}+\lambda x\right)<r_{0}$. Since $f\left(x_{0}+\lambda x\right)$ is continuous in $\lambda$, it follows from the Mean-value Theorem that there is a positive number $\lambda_{x}$ such that $f\left(x_{0}+\lambda_{x} x\right)=r_{0}$.

We shall show the uniqueness of $\lambda_{x}$. To show a contradiction, suppose that it is not unique, i. e., there are positive numbers $\lambda_{x}^{\prime}, \lambda_{x}^{\prime \prime}$, such that

$$
0<\lambda_{x}^{\prime}<\lambda_{x}^{\prime \prime} \text { and } f\left(x_{0}+\lambda_{x}^{\prime} x\right)=f\left(x_{0}+\lambda_{x}^{\prime \prime} x\right)=r_{0} .
$$

It is then true, by the convexity of $f$, that

$$
\begin{aligned}
r_{0} & =f\left(x_{0}+\lambda_{x}^{\prime} x\right) \\
& =f\left(\left(1-\frac{\lambda_{x}^{\prime}}{\lambda_{x}^{\prime \prime}}\right) x_{0}+\frac{\lambda_{x}^{\prime}}{\lambda_{x}^{\prime \prime}}\left(x_{0}+\lambda_{x}^{\prime \prime} x\right)\right) \\
& \leqq \\
& <\left(1-\frac{\lambda_{x}^{\prime}}{\lambda_{x}^{\prime \prime}}\right) f\left(x_{0}\right)+\frac{\lambda_{x}^{\prime}}{\lambda_{x}^{\prime \prime}} f\left(x_{0}+\lambda_{x}^{\prime \prime} x\right) \\
& <\left(1-\frac{\lambda_{x}^{\prime}}{\lambda_{x}^{\prime \prime}}\right) r_{0}+\frac{\lambda_{x}^{\prime}}{\lambda_{x}^{\prime \prime}} r_{0}=r_{0},
\end{aligned}
$$

since $0<1-\frac{\lambda_{x}^{\prime}}{\lambda_{x}^{\prime \prime}}<1$ and $f\left(x_{0}\right)<r_{0}$. This is a contradiction.

Q. E. D. 
LEMMA 3.3. Under Assumption 1, we have the following relations. For each vector $x(\neq 0) \in X$,

i) $f\left(x_{0}+x\right) \leqq r_{0}$ if and only if $\lambda_{x} \geqq 1$,

ii) $f\left(x_{0}+x\right) \geqq r_{0}$ if and only if $0<\lambda_{x} \leqq 1$.

Proof. i) "only if" part. For every $x(\neq 0)$ satisfying $f\left(x_{0}+x\right) \leqq r_{0}$, if we suppose that $0<\lambda_{x}<1$, then we obtain

$$
\begin{aligned}
r_{0} & =f\left(x_{0}+\lambda_{x} x\right) \\
& =f\left(\left(1-\lambda_{x}\right) x_{0}+\lambda_{x}\left(x_{0}+x\right)\right) \\
& \leqq\left(1-\lambda_{x}\right) f\left(x_{0}\right)+\lambda_{x} f\left(x_{0}+x\right)<r_{0},
\end{aligned}
$$

since $r_{0}>f\left(x_{0}\right)$ and $0<\lambda_{x}<1$. This is a contradiction.

"if" part. For each $x(\neq 0)$ satisfying $\lambda_{x} \geqq 1$, if we assume that $f\left(x_{0}+x\right)>r_{0}$, then we have

$$
\begin{aligned}
r_{0}<f\left(x_{0}+x\right) & =f\left(\left(1-\frac{1}{\lambda_{x}}\right) x_{0}+\frac{1}{\lambda_{x}}\left(x_{0}+\lambda_{x} x\right)\right) \\
& \leqq\left(1-\frac{1}{\lambda_{x}}\right) f\left(x_{0}\right)+\frac{1}{\lambda_{x}} f\left(x_{0}+\lambda_{x} x\right) \leqq r_{0} .
\end{aligned}
$$

This is a contradiction.

ii) "only if" part. For every $x$ satisfying $f\left(x_{0}+x\right) \geqq r_{0}$, if we assume that $\lambda_{x}>1$, then we obtain

$$
\begin{aligned}
r_{0} \leqq f\left(x_{0}+x\right) & =f\left(\left(1-\frac{1}{\lambda_{x}}\right) x_{0}+\frac{1}{\lambda_{x}}\left(x_{0}+\lambda_{x} x\right)\right) \\
& \leqq\left(1-\frac{1}{\lambda_{x}}\right) f\left(x_{0}\right)+\frac{1}{\lambda_{x}} f\left(x_{0}+\lambda_{x} x\right) \\
& <\left(1-\frac{1}{\lambda_{x}}\right) r_{0}+\frac{1}{\lambda_{x}} r_{0}=r_{0}
\end{aligned}
$$

since $0<1-\frac{1}{\lambda_{x}}<1$ and $f\left(x_{0}\right)<r_{0}$. This is a contradiction.

" if " part. Suppose that $f\left(x_{0}+x\right)<r_{0}$ for every $x$ satisfying $0<\lambda_{x} \leqq 1$. It is then valid that

$$
\begin{aligned}
r_{0} & =f\left(x_{0}+\lambda_{x} x\right) \\
& =f\left(\left(1-\lambda_{x}\right) x_{0}+\lambda_{x}\left(x_{0}+x\right)\right) \\
& \leqq\left(1-\lambda_{x}\right) f\left(x_{0}\right)+\lambda_{x} f\left(x_{0}+x\right) \\
& <\left(1-\lambda_{x}\right) r_{0}+\lambda_{x} r_{0}=r_{0} .
\end{aligned}
$$

This is a contradiction.

Q.E. D.

Lemma 3.4. Under Assumption $1, f$ is bounded below on the entire space $X$.

Proof. To show the contradiction, assume that $f$ is not bounded below on $X$, i. e., there exists a sequence $\left\{x_{n}\right\} \subset G_{r_{0}}$ such that $f\left(x_{n}\right) \rightarrow-\infty$ as $n \rightarrow \infty$. Without loss of generality, suppose that $f\left(x_{n}\right)<f\left(x_{0}\right)\left(<r_{0}\right)$ for all $n$. For each $x_{n}$ defined above, it follows from Lemmas 3.2 and 3.3 that there is a unique positive number $\lambda_{x_{0}-x_{n}}$ such that $f\left(x_{n}+\lambda_{x_{0}-x_{n}}\left(x_{0}-x_{n}\right)\right)=r_{0}$ and $\lambda_{x_{0}-x_{n}}>1$. If we suppose that $\lambda_{x_{0}-x_{n}} \rightarrow 1$ as $n \rightarrow \infty$, then we have 
and hence

$$
\begin{aligned}
\left\|x_{n}+\lambda_{x_{0}-x_{n}}\left(x_{0}-x_{n}\right)-x_{0}\right\| & =\left(\lambda_{x_{0}-x_{n}}-1\right)\left\|x_{0}-x_{n}\right\| \\
& \leqq\left(\lambda_{x_{0}-x_{n}}-1\right)\left(\left\|x_{0}\right\|+\left\|x_{n}\right\|\right) \\
& \leqq\left(\lambda_{x_{0}-x_{n}}-1\right) 2 b_{0} \longrightarrow 0 \text { as } n \rightarrow \infty,
\end{aligned}
$$

$$
x_{n}+\lambda_{x_{0}-x_{n}}\left(x_{0}-x_{n}\right) \longrightarrow x_{0} \quad \text { as } n \rightarrow \infty .
$$

Since $f$ is continuous by Lemma 3.1, it is clear that

$$
r_{0}=f\left(x_{n}+\lambda_{x_{0}-x_{n}}\left(x_{0}-x_{n}\right)\right) \underset{n \rightarrow \infty}{\longrightarrow} f\left(x_{0}\right)<r_{0} .
$$

This contradiction shows that there exist a positive number $\varepsilon_{1}$ and a subsequence $\left\{\lambda_{n}\right\}$ of $\left\{\lambda_{x_{0}-x_{n}}\right\}$ such that $\lambda_{n}>1+\varepsilon_{1}$ for all $n$. It is then true that

$$
\begin{aligned}
0<\frac{1}{\lambda_{n}} & <\frac{1}{1+\varepsilon_{1}}<1, \quad 1-\frac{1}{\lambda_{n}}>\frac{\varepsilon_{1}}{1+\varepsilon_{1}}>0, \\
f\left(x_{0}\right) & =f\left(\left(1-\frac{1}{\lambda_{n}}\right) x_{n}+\frac{1}{\lambda_{n}}\left(x_{n}+\lambda_{n}\left(x_{0}-x_{n}\right)\right)\right) \\
& \leqq \\
& \left(1-\frac{1}{\lambda_{n}}\right) f\left(x_{n}\right)+\frac{1}{\lambda_{n}} f\left(x_{n}+\lambda_{n}\left(x_{0}-x_{n}\right)\right) \\
& =\left(1-\frac{1}{\lambda_{n}}\right) f\left(x_{n}\right)+\frac{1}{\lambda_{n}} r_{0} .
\end{aligned}
$$

Since $f\left(x_{n}\right)<0$ for all sufficiently large $n$, it is easily verified that

$$
\begin{aligned}
f\left(x_{0}\right) & \leqq\left(1-\frac{1}{\lambda_{n}}\right) f\left(x_{n}\right)+\frac{1}{\lambda_{n}} r_{0} \\
& \leqq \frac{\varepsilon_{1}}{1+\varepsilon_{1}} f\left(x_{n}\right)+\left|r_{0}\right| \longrightarrow-\infty \quad \text { as } n \rightarrow \infty .
\end{aligned}
$$

This is a contradiction.

Q.E. D.

LEMma 3.5. Suppose that Assumption 1 holds. Let us define the real-valued function $p(x)$ on $X$ by

$$
p(x)=\left\{\begin{array}{lll}
\frac{1}{\lambda_{x}} & \text { for } & x \neq 0 \\
0 & \text { for } & x=0,
\end{array}\right.
$$

where $\lambda_{x}$ is stated in Lemma 3.2. Then, the function $p(x)$ is sublinear, i.e.,

$$
\begin{array}{ll}
p(\mu x)=\mu p(x) & \text { for all } \mu \geqq 0, \\
p(x+y) \leqq p(x)+p(y) & \text { for all } x, y \in X .
\end{array}
$$

PRoof. For every $\mu>0$ and every $x(\neq 0) \in X$, it follows from Lemma 3.2 that there are positive numbers $\lambda_{x}$ and $\lambda_{\mu x}$ such that

$$
f\left(x_{0}+\lambda_{x} x\right)=r_{0}=f\left(x_{0}+\lambda_{\mu x} \mu x\right) .
$$

By virtue of uniqueness of $\lambda_{x}$ for each $x$, we have $\lambda_{x}=\mu \lambda_{\mu x}$, and, therefore, it is valid that

$$
p(\mu x)=\frac{1}{\lambda_{\mu x}}=\frac{\mu}{\lambda_{x}}=\mu p(x) .
$$


Consequently, we can conclude that $p(x)$ is positively homogeneous, i. e.,

$$
p(\mu x)=\mu p(x) \quad \text { for all } \mu \geqq 0 \text { and all } x \in X .
$$

We shall show that

$$
p(x+y) \leqq p(x)+p(y) \quad \text { for every } \quad x, y \in X .
$$

If at least one of three vectors $x, y$, and $x+y$ is the origin, the inequality (3.1) holds since $p(x) \geqq 0$ for all $x \in X$, and $p(0)=0$. Now, assume that none of $x, y$ and $x+y$ is the origin. It is easily verified that

$$
\begin{gathered}
x_{0}+\frac{\lambda_{x} \lambda_{y}}{\lambda_{x}+\lambda_{y}}(x+y)=\frac{\lambda_{y}}{\lambda_{x}+\lambda_{y}}\left(x_{0}+\lambda_{x} x\right)+\frac{\lambda_{x}}{\lambda_{x}+\lambda_{y}}\left(x_{0}+\lambda_{y} y\right), \\
\begin{aligned}
f\left(x_{0}+\frac{\lambda_{x} \lambda_{y}}{\lambda_{x}+\lambda_{y}}(x+y)\right) & \leqq \frac{\lambda_{y}}{\lambda_{x}+\lambda_{y}} f\left(x_{0}+\lambda_{x} x\right)+\frac{\lambda_{x}}{\lambda_{x}+\lambda_{y}} f\left(x_{0}+\lambda_{y} y\right) \\
& =\frac{\lambda_{y}}{\lambda_{x}+\lambda_{y}} r_{0}+\frac{\lambda_{x}}{\lambda_{x}+\lambda_{y}} r_{0}=r_{0} .
\end{aligned}
\end{gathered}
$$

It follows from Lemma 3.3 that

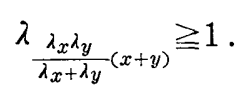

The positive homogeneity of $p(x)$ implies

$$
1 \leqq \lambda_{\frac{\lambda_{x} \lambda_{y}}{\lambda_{x}+\lambda_{y}}(x+y)}=\frac{\lambda_{x}+\lambda_{y}}{\lambda_{x} \lambda_{y}} \lambda_{x+y},
$$

or

$$
\frac{1}{\lambda_{x+y}} \leqq \frac{1}{\lambda_{x}}+\frac{1}{\lambda_{y}}
$$

and hence $p(x+y) \leqq p(x)+p(y)$.

Q.E. D.

LEMMA 3.6. Suppose that Assumption 1 holds. It is then valid that

i) $f\left(x_{0}+x\right) \leqq r_{0}$ if and only if $0 \leqq p(x) \leqq 1$,

ii) $f\left(x_{0}+x\right) \geqq r_{0}$ if and only if $p(x) \geqq 1$.

Note that $x$ may be the origin.

Proof. This is an immediate consequence of Lemma 3.3 and the definition of $p(x)$.

Q. E. D.

REMARK. The above $p(x)$ is compared with the Minkovski functional of the set $\left\{x \in X \mid f(x) \leqq r_{0}\right\}-x_{0}$.

Lemma 3.7. Suppose that Assumption 1 holds. Then $p(x)$ is continuous on $X$.

Proof. It follows from Lemma 3.6 that $\{x \in X \mid p(x)<1\}=G_{r_{0}}-x_{0}$. Since $G_{r_{0}}$ is a non-empty open set, $p(x)$ is continuous on $X$.

LEMma 3.8. Suppose that Assumption 1 holds. If we define the functional $\bar{p}(x)$ by

then we have

$$
\tilde{p}(x)=p(x)\left(r_{0}-f\left(x_{0}\right)\right)+f\left(x_{0}\right) \quad \text { for all } \quad x \in X,
$$

i) $f\left(x_{0}+x\right) \leqq r_{0}$ implies $\bar{p}(x) \geqq f\left(x_{0}+x\right)$,

ii) $f\left(x_{0}+x\right) \geqq r_{0}$ implies $\bar{p}(x) \leqq f\left(x_{0}+x\right)$ for all $x \in X$. 
Proof. i) If $x=0$, then it holds by the definition of $\bar{p}(x)$. Suppose that $x \neq 0$. It is easily verified, by Lemma 3.3 , that

$$
\begin{aligned}
f\left(x_{0}+x\right) & =f\left(\left(1-\frac{1}{\lambda_{x}}\right) x_{0}+\frac{1}{\lambda_{x}}\left(x_{0}+\lambda_{x} x\right)\right) \\
& \leqq\left(1-\frac{1}{\lambda_{x}}\right) f\left(x_{0}\right)+\frac{1}{\lambda_{x}} f\left(x_{0}+\lambda_{x} x\right) \\
& =(1-p(x)) f\left(x_{0}\right)+p(x) r_{0}=\bar{p}(x),
\end{aligned}
$$

since $\lambda_{x} \geqq 1$ whenever $f\left(x_{0}+x\right) \leqq r_{0}$.

ii) It follows from Lemma 3.3 that $0<\lambda_{x} \leqq 1$ whenever $f\left(x_{0}+x\right) \geqq r_{0}$. It is then true that

$$
\begin{aligned}
f\left(x_{0}+\lambda_{x} x\right) & =f\left(\left(1-\lambda_{x}\right) x_{0}+\lambda_{x}\left(x_{0}+x\right)\right) \\
& \leqq\left(1-\lambda_{x}\right) f\left(x_{0}\right)+\lambda_{x} f\left(x_{0}+x\right)
\end{aligned}
$$

and hence

$$
f\left(x_{0}+x\right) \geqq r_{0} p(x)-(p(x)-1) f\left(x_{0}\right)=\bar{p}(x) .
$$

Q.E.D.

Lemma 3.9. Under Assumption 1, for every real number $r$ such that the set $G_{r} \equiv\{x \in X \mid f(x)<r\}$ is non-empty, the set $G_{r}$ is bounded and open in $X$.

Proof. The openness immediately follows from Lemma 3.1. It is true, by Assumption 1 , that there is a positive number $b_{0}$ such that $\|x\| \leqq b_{0}$ for all $x \in G_{r_{0}}$. For each $r \leqq r_{0}, G_{r}$ is bounded since $G_{r_{0}}$ is bounded.

For every $r>r_{0}$, we shall show that

$$
P_{r} \equiv\left\{x \in X \mid \bar{p}\left(x-x_{0}\right)<r\right\} \supset G_{r},
$$

where the functional $\bar{p}(x)$ is defined in Lemma 3.8. For every $x \in G_{r}$ satisfying $f(x) \leqq r_{0}$, it follows from Lemma 3.6 that $p\left(x-x_{0}\right) \leqq 1$, so that

$$
\begin{aligned}
\bar{p}\left(x-x_{0}\right) & =p\left(x-x_{0}\right)\left(r_{0}-f\left(x_{0}\right)\right)+f\left(x_{0}\right) \\
& \leqq\left(r_{0}-f\left(x_{0}\right)\right)+f\left(x_{0}\right)=r_{0}<r,
\end{aligned}
$$

and hence $x \in P_{r}$. For every $x \in G_{r}$ such that $r_{0}<f(x)<r$, we have, by Lemma 3.8, $\bar{p}\left(x-x_{0}\right) \leqq f(x)<r$, which implies that $x \in P_{r}$. Therefore, it suffices to show that the set $P_{r}$ is bounded. For each $x \in P_{r}$, we have

$$
p\left(x-x_{0}\right)<\frac{r-f\left(x_{0}\right)}{r_{0}-f\left(x_{0}\right)}
$$

or

$$
p\left(\frac{r_{0}-f\left(x_{0}\right)}{r-f\left(x_{0}\right)}\left(x-x_{0}\right)\right)<1 .
$$

It follows from Lemma 3.6 that

so that

$$
f\left(x_{0}+\frac{r_{0}-f\left(x_{0}\right)}{r-f\left(x_{0}\right)}\left(x-x_{0}\right)\right)<r_{0},
$$

$$
\left\|x_{0}+\frac{r_{0}-f\left(x_{0}\right)}{r-f\left(x_{0}\right)}\left(x-x_{0}\right)\right\| \leqq b_{0},
$$


and hence

$$
\frac{r_{0}-f\left(x_{0}\right)}{r-f\left(x_{0}\right)}\|x\| \leqq b_{0}+\left(1-\frac{r_{0}-f\left(x_{0}\right)}{r-f\left(x_{0}\right)}\right)\left\|x_{0}\right\|
$$

which implies the boundedness of $P_{r}$.

Q.E.D.

Under Assumption 1, there is a real number $d_{0}$ such that $d_{0}=\inf _{x \in X} f(x)$ since the convex function $f(x)$ is bounded below by Lemma 3.4. For every $d>d_{0}$, the set $G_{d}$ is a non-empty, bounded open set in $X$ by Lemma 3.9. Moreover, it is valid, by Lemma 3.2 , that to every $x \in X(x \neq 0)$, there corresponds a unique positive number $\lambda_{x}$ such that $f\left(x_{0}+\lambda_{x} x\right)=d$, where the vector $x_{0}$ satisfies $f\left(x_{0}\right)<d$. Similarly, for each $d>d$, there is a unique positive number $\tilde{\lambda}_{x}$ such that $f\left(x_{0}+\tilde{\lambda}_{x} x\right)=\tilde{d}$. Then, we can state the following lemmas :

LEMMA 3.10. Under Assumption $1, \tilde{\lambda}_{x}>\lambda_{x}$ for all $x \in X(x \neq 0)$.

Proof. To show the contradiction, suppose that $0<\tilde{\lambda}_{x} \leqq \lambda_{x}$. It is then true that

$$
\begin{aligned}
\tilde{d} & =f\left(x_{0}+\tilde{\lambda}_{x} x\right) \\
& =f\left(\left(1-\frac{\tilde{\lambda}_{x}}{\lambda_{x}}\right) x_{0}+\frac{\tilde{\lambda}_{x}}{\lambda_{x}}\left(x_{0}+\lambda_{x} x\right)\right) \\
& \leqq\left(1-\frac{\tilde{\lambda}_{x}}{\lambda_{x}}\right) f\left(x_{0}\right)+\frac{\tilde{\lambda}_{x}}{\lambda_{x}} f\left(x_{0}+\lambda_{x} x\right) \leqq d .
\end{aligned}
$$

This contradicts to the fact that $\tilde{d}>d$.

Q.E.D.

LEMMA 3.11. Suppose that Assumption 1 holds. Then, there is a positive number $\tilde{c}>0$ such that $\tilde{c}>\sup _{\|x\|=1} \tilde{\lambda}_{x}$.

Proof. It follows from Lemma 3.9 that $G_{\tilde{d}}$ is bounded in $X$ and hence so is the set $F_{\widetilde{d}} \equiv\{x \in X \mid f(x) \leqq \tilde{d}\}$, i. e., there is a positive number $\tilde{b}$ such that

$$
\|x\| \leqq \tilde{b} \quad \text { for all } \quad x \in F_{\widetilde{a}} .
$$

To show the contradiction, suppose that there is a sequence of vectors $\left\{x_{n}\right\}$ such that $\left\|x_{n}\right\|=1$ for all $n=1,2, \cdots$, and $\tilde{\lambda}_{x_{n}} \rightarrow \infty$ as $n \rightarrow \infty$. Since

$$
\left\|x_{0}+\tilde{\lambda}_{x_{n}} x_{n}\right\| \geqq\left\|\tilde{\lambda}_{x_{n}} x_{n}\right\|-\left\|x_{0}\right\|=\tilde{\lambda}_{x_{n}}-\left\|x_{0}\right\|,
$$

there is a sufficiently large positive integer $N$ such that $\left\|x_{0}+\tilde{\lambda}_{x_{N}} x_{N}\right\|>\tilde{b}$. Then, (3.3) implies that $f\left(x_{0}+\tilde{\lambda}_{x_{N}} x_{N}\right)>\tilde{d}$, which contradicts to the fact that $\tilde{d}=f\left(x_{0}+\tilde{\lambda}_{x_{N}} x_{N}\right)$.

LEMMA 3.12. Let a real-valued function $f$ defined over a linear space $X$ be convex. Then, it is valid that

$$
f(y+\varepsilon(y-x)) \geqq f(y)+\varepsilon(f(y)-f(x)) \quad \text { for all } x, y \in X \text { and all } \varepsilon>0 .
$$

Proof. It is clear that

$$
\begin{aligned}
& 0<\frac{\varepsilon}{1+\varepsilon}, \quad \frac{1}{1+\varepsilon}<1, \\
& y=\frac{\varepsilon}{1+\varepsilon} x+\frac{1}{1+\varepsilon}(y+\varepsilon(y-x)) \quad \text { for all } \varepsilon>0 \text { and all } x, y \in X .
\end{aligned}
$$


We obtain

$$
f(y) \leqq \frac{\varepsilon}{1+\varepsilon} f(x)+\frac{1}{1+\varepsilon} f(y+\varepsilon(y-x)),
$$

which implies

$$
f(y+\varepsilon(y-x)) \geqq f(y)+\varepsilon(f(y)-f(x)) .
$$

We now introduce another assumption for $f$.

Q. E. D.

Assumption 2. Let us define the set $S_{t}$ in a normed linear space $X$ by $S_{t} \equiv$ $\{x \in X \mid\|x\| \leqq t\}$ for each $t>0$. The function $f: X \rightarrow R^{1}$ is bounded above on $S_{t}$ for all $t>0$.

LEMma 3.13. Suppose that Assumptions 1 and 2 hold. There is a positive number $\nu_{0}$ such that

$$
\inf _{\|x\|=1}\left(\tilde{\lambda}_{x}-\lambda_{x}\right) \geqq \nu_{0} \quad \text { whenever } \quad \tilde{d}>d>d_{0} .
$$

ProOF. First of all, note that $\tilde{\lambda}_{x}>\lambda_{x}$ for all $x(\neq 0) \in X$. To show the contradiction, suppose that $\inf _{\|x\|=1}\left(\tilde{\lambda}_{x}-\lambda_{x}\right)=0$. Then, there is a sequence $\left\{x_{n}\right\} \subset X$ such that $\left\|x_{n}\right\|=1$ for all $n=1,2, \cdots$, and $\tilde{\lambda}_{x_{n}}-\lambda_{x_{n}} \rightarrow 0$ as $n \rightarrow \infty$. It follows from Lemma 3.12 that for every positive number $\varepsilon$, we have

$$
\begin{aligned}
f\left(x_{0}+\lambda_{x} x+(1+\varepsilon)\left(\tilde{\lambda}_{x}-\lambda_{x}\right) x\right) \\
\quad \geqq(1+\varepsilon)\left[f\left(x_{0}+\tilde{\lambda}_{x} x\right)-f\left(x_{0}+\lambda_{x} x\right)\right]+f\left(x_{0}+\lambda_{x} x\right) \\
\quad=(1+\varepsilon)(\tilde{d}-d)+d=\tilde{d}+\varepsilon(\tilde{d}-d) \quad \text { for each } \quad x(\neq 0) \in X .
\end{aligned}
$$

If we define the positive numbers $\varepsilon_{n}, n=1,2, \cdots$, by

$$
\varepsilon_{n} \equiv \frac{\tilde{c}-\tilde{\lambda}_{x_{n}}}{\tilde{\lambda}_{x_{n}}-\lambda_{x_{n}}},
$$

then $\varepsilon_{n} \rightarrow \infty$ as $n \rightarrow \infty$. It is evident that

$$
\begin{aligned}
f\left(x_{0}+\tilde{c} x_{n}\right) & =f\left(x_{0}+\lambda_{x_{n}} x_{n}+\left(1+\frac{\tilde{c}-\tilde{\lambda}_{x_{n}}}{\tilde{\lambda}_{x_{n}}-\lambda_{x_{n}}}\right)\left(\tilde{\lambda}_{x_{n}}-\lambda_{x_{n}}\right) x_{n}\right) \\
& \geqq \tilde{d}+\varepsilon_{n}(\tilde{d}-d) \longrightarrow \infty \quad \text { as } n \rightarrow \infty .
\end{aligned}
$$

Since $\left\|x_{0}+\tilde{c} x_{n}\right\| \leqq\left\|x_{0}\right\|+\tilde{c}$, it is valid, by Assumption 2, that there is a positive number $M$ such that $f\left(x_{0}+\tilde{c} x_{n}\right) \leqq M$ for all $n=1,2, \cdots$, which is a contradiction.

Q. E. D.

LEMMA 3.14. Let the real-valued function $f(x)$ on $X$ be a sublinear function which satisfies

$$
\begin{aligned}
& f(x) \geqq 0 \quad \text { for all } \quad x \in X, \\
& f(x)=0 \text { if and only if } x=0 .
\end{aligned}
$$

Then, the function $f(x)$ is uniformly convex if and only if for an arbitrary positive number $\varepsilon$, there exists a positive number $\delta=\delta(\varepsilon)$ such that

$$
f(x) \leqq 1, \quad f(y) \leqq 1, \quad\|x-y\| \geqq \varepsilon \quad \text { implies } \quad f\left(\frac{x+y}{2}\right) \leqq 1-\delta .
$$


PROOF. It is clear that every uniformly convex function $f(x)$ satisfies the condition (3.4). We shall show the contrary. There is no vector $x$ satisfying $f(x)<d$ for every $d<0$. If $d=0$, then there is no pair of vectors $x$ and $y$ such that $f(x) \leqq 0, f(y) \leqq 0$, and $\|x-y\| \geqq \varepsilon$ for any $\varepsilon>0$. For every $d>0$ and every $\varepsilon>0$, let the vectors $x$ and $y$ satisfy $f(x) \leqq d, f(y) \leqq d$, and $\|x-y\| \geqq \varepsilon$, and hence we have

$$
f\left(\frac{x}{d}\right) \leqq 1, \quad f\left(\frac{y}{d}\right) \leqq 1 \quad \text { and } \quad\left\|\frac{x}{d}-\frac{y}{d}\right\| \geqq \frac{\varepsilon}{d} .
$$

It is valid, by (3.4), that there is a positive number $\delta\left(\frac{\varepsilon}{d}\right)$ such that

$$
f\left(\frac{x+y}{2}\right) \leqq d-d \delta\left(\frac{\varepsilon}{d}\right) .
$$

Consequently, the function $f$ is uniformly convex with $\delta(d, \varepsilon) \equiv d \delta\left(\frac{\varepsilon}{d}\right)>0 . \quad$ Q. E. D.

Lemma 3.15. Suppose that Assumptions 1 and 2 hold. For arbitrary positive number $\eta_{1}$ and $\eta_{2}$ satisfying $0<\eta_{1} \leqq \eta_{2}$, there is a positive number $\nu_{1}$ such that

$$
\inf _{\eta_{1} \leqq\|x\| \leqq \eta_{2}}\left(\tilde{\lambda}_{x}-\lambda_{x}\right) \geqq \nu_{1} .
$$

PROOF. It follows from Lemma 3.13 that there is a positive number $\nu_{0}$ such that $\inf _{\|x\|=1}\left(\tilde{\lambda}_{x}-\lambda_{x}\right) \geqq \nu_{0}$ we shall show that

$$
\inf _{\eta_{1} \leqq\|x\| \leqq \eta_{2}}\left(\tilde{\lambda}_{x}-\lambda_{x}\right)=\inf _{\eta_{1} \leqq \eta_{1} \leqq \eta_{2}} \inf _{\|x\|=\eta}\left(\tilde{\lambda}_{x}-\lambda_{x}\right) .
$$

If we define the sets $E$ and $E_{\eta}$ by

$$
\begin{aligned}
& E \equiv\left\{x \in X \mid \eta_{1} \leqq\|x\| \leqq \eta_{2}\right\}, \\
& E_{\eta} \equiv\{x \in X \mid\|x\|=\eta\} \quad \text { for every } \quad \eta>0,
\end{aligned}
$$

then it is immediately seen that

$$
E=\bigcup_{\eta_{1} \leqq \eta \leqq \eta_{2}} E_{r_{1}},
$$

so that

$$
\inf _{x \in E}\left(\tilde{\lambda}_{x}-\lambda_{x}\right) \leqq \inf _{\eta_{1} \leqq \eta \leqq \eta_{2}} \inf _{x \in E \eta}\left(\tilde{\lambda}_{x}-\lambda_{x}\right) .
$$

On the other hand, for every vector $x \in E$, there is a positive number $\eta, \eta_{1} \leqq \eta \leqq \eta_{2}$, such that $\|x\|=\eta$, and hence we have

$$
\inf _{\eta_{1} \leqq \eta \leqq \eta_{2}} \inf _{x \in E \eta}\left(\tilde{\lambda}_{x}-\lambda_{x}\right) \leqq \tilde{\lambda}_{x}-\lambda_{x}
$$

This establishes the equality (3.5).

Note that, by Lemma 3.5 ,

We then obtain

$$
\tilde{\lambda}_{\eta x}=\frac{\tilde{\lambda}_{x}}{\eta}, \quad \lambda_{\eta x}=\frac{\lambda_{x}}{\eta} \quad \text { for all } \quad \eta>0
$$

$$
\begin{aligned}
\inf _{\eta_{1} \leqq\|x\| \leqq \eta_{2}}\left(\tilde{\lambda}_{x}-\lambda_{x}\right) & =\inf _{\eta_{1} \leqq \eta \leqq \eta_{2}} \inf _{\|x\|=\eta}\left(\tilde{\lambda}_{x}-\lambda_{x}\right) \\
& =\inf _{\eta_{1} \leqq \eta \leqq \eta_{2}} \inf _{x \|=1}\left(\tilde{\lambda}_{\eta_{x}}-\lambda_{\eta_{x}}\right)
\end{aligned}
$$




$$
=\inf _{\eta_{1} \leqq \eta \leqq \eta_{2}} \frac{1}{\eta} \inf _{\|x\|=1}\left(\tilde{\lambda}_{x}-\lambda_{x}\right) \geqq \inf _{\eta_{1} \leqq \eta \leqq \eta_{2}} \frac{\nu_{0}}{\eta} \geqq \frac{\nu_{0}}{\eta_{2}} .
$$

Therefore, the condition of Lemma 3.15 holds with $\nu_{1} \equiv \nu_{0} / \eta_{2}$.

Q.E.D.

Now we shall review the preceding arguments. Let $f(x)$ be a convex function defined on a normed linear space $X$. Suppose that Assumptions 1 and 2 hold. Since $f$ is bounded below by Lemma 3.4, there is a real number $d_{0}$ such that $d_{0}=\inf _{x \in X} f(x)$. It is valid, by Lemma 3.9, that the set $G_{d}$ is a non-empty, bounded open set in $X$ for every $d>d_{0}$. It then follows from Lemma 3.2 that for each $d>d_{0}$, and every $x(\neq 0) \in X$, there exists a unique positive number $\lambda_{x}$ such that $f\left(x_{0}+\lambda_{x} x\right)=d$, where $f\left(x_{0}\right)<d$. Let $p(x)$ be the functional defined in Lemma 3.5. Then, we can describe the following proposition :

Proposition 3.1. Under the conditions stated above, if $f(x)$ is uniformly convex, then $p(x)$ is also uniformly convex.

Proof. It follows from Lemma 3.5 that $p(x)$ is sublinear on $X$ and

$$
\begin{aligned}
& p(x) \geqq 0 \quad \text { for all } \quad x \in X, \\
& p(x)=0 \quad \text { if and only if } \quad x=0 .
\end{aligned}
$$

By virtue of Lemma 3.14, it is sufficient to show that for every positive number $\varepsilon$ there is a positive number $\delta=\delta(\varepsilon)$ such that

$$
p(x) \leqq 1, \quad p(y) \leqq 1 \text { and }\|x-y\| \geqq \varepsilon \text { implies } p\left(\frac{x+y}{2}\right) \leqq 1-\delta,
$$

in order to point out the uniform convexity of $p(x)$. To show the contradiction, suppose that there is a positive number $\varepsilon_{0}$ such that for every positive number $\delta$ there exist vectors $x_{\delta}$ and $y_{\dot{o}}$ satisfying

$$
p\left(x_{\tilde{\delta}}\right) \leqq 1, \quad p\left(y_{\tilde{\delta}}\right) \leqq 1, \quad\left\|x_{\dot{\delta}}-y_{\hat{o}}\right\| \geqq \varepsilon_{0}, \quad \text { and } \quad 1 \geqq p\left(\frac{x_{\dot{\delta}}+y_{\tilde{o}}}{2}\right)>1-\delta .
$$

Since $p(x)$ is continuous and since the origin is an interior point of the set $\{x \in X \mid p(x)$ $\left.\leqq \frac{1}{2}\right\}$, there is a positive number $\eta_{1}$ such that

$$
\left\|\frac{x_{\delta}+y_{\delta}}{2}\right\| \geqq \eta_{1} \quad \text { whenever } \quad 0<\delta<\frac{1}{2} .
$$

It follows from Lemma 3.6 that

$$
f\left(x_{0}+\frac{x_{\delta}+y_{\delta}}{2}\right) \leqq d \quad \text { whenever } \quad 0<\delta<\frac{1}{2} .
$$

It is true, by Lemma 3.9 , that the set $F_{d} \equiv\{x \in X \mid f(x) \leqq d\}$ is bounded in $X$ and hence there is a positive number $\eta_{2}$ satisfying

$$
\eta_{2} \geqq\left\|\frac{x_{\delta}+y_{\delta}}{2}\right\| \geqq \eta_{1} \quad \text { whenever } \quad 0<\delta<\frac{1}{2} .
$$

Let us fix an arbitrary real number $d$ satisfying $d>d$. Then, for each vector $x(\neq 0) \in X$, there exists a unique positive number $\tilde{\lambda}_{x}$ such that $f\left(x_{0}+\tilde{\lambda}_{x} x\right)=\tilde{d}$. It is then valid, by virtue of Lemma 3.15 , that there is a positive number $\nu_{1}$ such that 
which implies

$$
\inf _{\eta_{1} \leqq\|x\| \leqq \eta_{2}}\left(\tilde{\lambda}_{x}-\lambda_{x}\right) \geqq \nu_{1},
$$

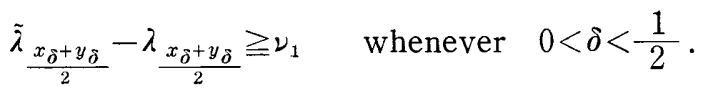

Since $f$ is uniformly convex, for the real numbers $d$ and $\varepsilon_{0}>0$, there is a positive number $\delta\left(d, \varepsilon_{0}\right)$ such that

$$
f(x) \leqq d, \quad f(y) \leqq d, \quad\|x-y\| \geqq \varepsilon_{0} \quad \text { implies } \quad f\left(\frac{x+y}{2}\right) \leqq d-\delta\left(d, \varepsilon_{0}\right) .
$$

It is then true, by Lemma 3.6 , that $f\left(x_{0}+x_{\tilde{o}}\right) \leqq d$ and $f\left(x_{0}+y_{\bar{o}}\right) \leqq d$, so that

$$
f\left(x_{0}+\frac{x_{\tilde{\delta}}+y_{\tilde{\delta}}}{2}\right)=f\left(\frac{\left(x_{0}+x_{\tilde{\delta}}\right)+\left(x_{0}+y_{\tilde{\delta}}\right)}{2}\right) \leqq d-\delta\left(d, \varepsilon_{0}\right)<d,
$$

which implies

$$
p\left(\frac{x_{\tilde{\delta}}+y_{\tilde{o}}}{2}\right)<1 \text { or } \frac{1}{1-\delta}>\lambda_{\frac{x_{\tilde{\delta}}+y_{\tilde{o}}}{2}}>1 \quad \text { whenever } \quad 0<\delta<\frac{1}{2} .
$$

It is easily seen, by Lemma 3.12 , that

$$
\begin{aligned}
& f\left(x_{0}+\lambda_{\frac{x_{\tilde{\delta}}+y_{\tilde{\delta}}}{2}} \frac{x_{\delta}+y_{\tilde{\delta}}}{2}+\varepsilon\left(\lambda_{\frac{x_{\tilde{\delta}}+y_{\tilde{\delta}}}{2}}-\tilde{\lambda}_{\frac{x_{\tilde{\delta}}+y_{\tilde{\delta}}}{2}}\right) \frac{x_{\tilde{\delta}}+y_{\tilde{\delta}}}{2}\right) \\
& \quad \geqq f\left(x_{0}+\lambda_{\frac{x_{\delta^{+}+y_{\delta}}}{2}} \frac{x_{\tilde{\delta}}+y_{\tilde{\delta}}}{2}\right)+\varepsilon\left(f\left(x_{0}+\lambda_{\frac{x_{\tilde{\delta}}+y_{\tilde{\delta}}}{2}} \frac{x_{\delta}+y_{\tilde{\delta}}}{2}\right)-f\left(x_{0}+\tilde{\lambda}_{\frac{x_{\tilde{\delta}}+y_{\tilde{\delta}}}{2}} \frac{x_{\delta}+y_{\tilde{\delta}}}{2}\right)\right) \\
& \quad=d+\varepsilon(d-\tilde{d}) \quad \text { for every } \quad \varepsilon>0 .
\end{aligned}
$$

If we set

$$
\varepsilon \equiv \frac{1-\lambda_{x_{\tilde{\delta}}+y_{\tilde{o}}}}{2},
$$

then we have

$$
f\left(x_{0}+\frac{x_{\tilde{\delta}}+y_{\tilde{\delta}}}{2}\right) \geqq d+\frac{\lambda_{x_{\tilde{\delta}}+y_{\tilde{\delta}}}-1}{\tilde{\lambda}_{\frac{x_{\tilde{\delta}}+y_{\tilde{\delta}}}{2}}-\lambda_{\frac{x_{\tilde{\delta}}+y_{\tilde{\delta}}}{2}}}(d-\tilde{d}) .
$$

It is then valid, by (3.7), that

$$
f\left(x_{0}+\frac{x_{\tilde{\delta}}+y_{\delta}}{2}\right) \geqq d+\frac{\frac{\lambda x_{\delta^{+} y_{\tilde{\delta}}}}{2}-1}{\nu_{1}}(d-\tilde{d}) \quad \text { whenever } \quad 0<\delta<\frac{1}{2} .
$$

For the positive number $\delta\left(d, \varepsilon_{0}\right)$, there is a positive number $\delta_{0}, 0<\delta_{0}<\frac{1}{2}$ such that

$$
0<\frac{\frac{\lambda_{x_{\delta_{0}}+y_{\delta_{0}}}}{2}-1}{\nu_{1}}(\tilde{d}-d)<\delta\left(d, \varepsilon_{0}\right) \text {, }
$$

which implies

$$
f\left(x_{0}+\frac{x_{\delta_{0}}+y_{\delta_{0}}}{2}\right)>d-\delta\left(d, \varepsilon_{0}\right) .
$$


This contradicts to (3.6).

Q.E.D.

Proposition 3.2. Let $f$ be uniformly convex and suppose that Assumptions 1 and 2 hold. If we set $F_{d} \equiv\{x \in X \mid f(x) \leqq d\}$ for every $d>d_{0}$, then for each positive number $\varepsilon$, there is a positive number $\delta=\delta(\varepsilon)$ such that

$$
\text { conuex set } A \subset F_{d+o} \cap F_{d}{ }^{c}, \quad x, y \in A \text { implies }\|x-y\|<\varepsilon,
$$

where $F_{d}{ }^{c}$ denotes the complement of $F_{d}$.

Proof. Note that the functional $p(x)$ is uniformly convex by Proposition 3.1 . To show the contradiction, suppose that there is a positive number $\varepsilon_{0}$ such that for every $\delta>0$, there exist the convex set $A_{\delta}$ and the vectors $x_{\delta}, y_{\delta} \in A_{\delta}$ satisfying

$$
A_{\hat{\delta}} \subset F_{d+\hat{o}} \cap F_{d}{ }^{c}, \quad x_{\hat{o}}, y_{\hat{o}} \in A \text { and }\left\|x_{\tilde{\delta}}-y_{\hat{o}}\right\| \geqq \varepsilon_{0} .
$$

It is then evident, by Lemma 3.6 , that $p\left(x_{\tilde{\delta}}-x_{0}\right)>1$ and $p\left(y_{\tilde{o}}-x_{0}\right)>1$. It follows from Lemma 3.8 that

$$
\begin{aligned}
& p\left(x_{\delta}-x_{0}\right)\left(d-f\left(x_{0}\right)\right)+f\left(x_{0}\right) \leqq f\left(x_{\tilde{\delta}}\right) \leqq d+\delta, \\
& p\left(y_{\tilde{o}}-x_{0}\right)\left(d-f\left(x_{0}\right)\right)+f\left(x_{0}\right) \leqq f\left(y_{\tilde{\delta}}\right) \leqq d+\delta
\end{aligned}
$$

and hence

$$
p\left(x_{\tilde{\delta}}-x_{0}\right) \leqq d(\delta) \text { and } p\left(y_{\tilde{o}}-x_{0}\right) \leqq d(\delta),
$$

where $d(\delta) \equiv \frac{d+\delta-f\left(x_{0}\right)}{d-f\left(x_{0}\right)}>1$. It is true that

$$
\left\|\frac{x_{\delta}-x_{0}}{d(\delta)}-\frac{y_{\delta}-x_{0}}{d(\delta)}\right\|=\frac{\left\|x_{\delta}-y_{\delta}\right\|}{d(\delta)} \geqq \frac{\varepsilon_{0}}{d(\delta)} \geqq \frac{\varepsilon_{0}}{d(1)}, \quad \text { for all } \quad \delta, 0<\delta<1,
$$

and

$$
p\left(\frac{x_{\tilde{\delta}}-x_{0}}{d(\delta)}\right) \leqq 1 \quad \text { and } \quad p\left(\frac{y_{\delta}-x_{0}}{d(\delta)}\right) \leqq 1 \quad \text { for every } \quad \delta, 0<\delta<1,
$$

which implies the existence of a positive number $\mu_{0}$ such that

$$
p\left(\frac{\frac{x_{\delta}+x_{0}}{d(\delta)}+\frac{y_{\delta}-x_{0}}{d(\delta)}}{2}\right) \leqq 1-\mu_{0},
$$

or

$$
p\left(\frac{x_{\delta}+y_{\delta}}{2}-x_{0}\right) \leqq d(\delta)\left(1-\mu_{0}\right) \quad \text { whenever } \quad 0<\delta<1,
$$

since $p(x)$ is uniformly convex. It is valid that there is a sufficiently small number $\delta_{0}, 0<\delta_{0}<1$, satisfying

$$
p\left(\frac{x_{\delta_{0}}+y_{\delta_{0}}}{2}-x_{0}\right) \leqq d\left(\delta_{0}\right)\left(1-\mu_{0}\right)<1
$$

which implies

$$
f\left(\frac{x_{\delta_{0}}+y_{\delta_{0}}}{2}\right)>d
$$

This contradicts to the fact that

$$
\frac{x_{\delta_{0}}+y_{\delta_{0}}}{2} \in A_{\delta_{0}} \subset F_{d+\delta_{0}} \cap F_{d}{ }^{c} .
$$

Q.E. D. 


\section{$\S 4$. Convergent minimizing sequences.}

In this section, we shall observe that the uniform convexity leads to the convergence of minimizing sequence and that it assures the existence and uniqueness of minimum.

Let $X$ be a normed linear space and let $f$ be a real-valued function defined over $X$. We shall set up two assumptions.

Assumption 3. For every positive number $\varepsilon$, there is a vector $x_{\varepsilon} \in X$ such that $f\left(x_{\varepsilon}\right)<\inf _{\|x\|=1} f\left(x_{\varepsilon}+\varepsilon x\right)$.

Assumption 4. The function $f$ is bounded above on the set $\{x \in X \mid\|x\|=t\}$ for every $t>0$.

PROPOSITION 4.1. Let $f$ be a real-valued convex function defined on a normed linear space $X$. If we suppose that Assumption 4 holds, then Assumption 3 is equivalent to the following condition:

(*) For every positive number $\varepsilon$, there exists a real number $d\left(>\inf _{x \in X} f(x)\right)$ such that $f(x) \leqq d$ and $f(y) \leqq d$ imply $\|x-y\|<\varepsilon$.

Proof. First of all, we shall show that Assumption 3 implies the condition $(*)$. Since $f$ is bounded below on $X$, by Lemma 3.4, then there is a real number $d_{0}$ such that $d_{0}=\inf _{x \in X} f(x)$. Assumption 3 assures that for every $\varepsilon>0$, there exists a vector $x_{\varepsilon / 2} \in X$ such that $f\left(x_{\varepsilon / 2}\right)<\inf _{\|x\|=1} f\left(x_{\varepsilon / 2}+\frac{\varepsilon}{2} x\right)$. Let $d$ be the number $\inf _{\|x\|=1} f\left(x_{\varepsilon / 2}+\frac{\varepsilon}{2} x\right)$. For each vector $x \in X$ satisfying $\|x\|>1$, we have

$$
\begin{aligned}
d & \leqq f\left(x_{\varepsilon / 2}+\frac{\varepsilon}{2} \frac{x}{\|x\|}\right) \\
& \leqq\left(1-\frac{1}{\|x\|}\right) f\left(x_{\varepsilon / 2}\right)+\frac{1}{\|x\|} f\left(x_{\varepsilon / 2}+\frac{\varepsilon}{2} x\right),
\end{aligned}
$$

and hence $f\left(x_{\varepsilon / 2}+\frac{\varepsilon}{2} x\right)>d$. Consequently, it is valid that

$$
f\left(x_{\varepsilon / 2}+\frac{\varepsilon}{2} x\right) \leqq d \quad \text { implies } \quad\|x\| \leqq 1 .
$$

For every $x, y \in X$ satisfying $f(x) \leqq d$ and $f(y) \leqq d$, we obtain

$$
\left\|x-x_{\varepsilon / 2}\right\| \leqq \frac{\varepsilon}{2} \text { and }\left\|y-x_{\varepsilon / 2}\right\| \leqq \frac{\varepsilon}{2},
$$

so that $\|x-y\| \leqq \varepsilon$, which implies that the condition (*) holds.

Conversely, we shall show that the condition (*) implies Assumption 3. We shall see that this case holds without convexity of $f$ although it is assumed that $f$ is convex. It is then noted that Assumption 3 is weaker than the condition (*) in this sense. There are following two cases:

Case 1. Suppose that $f$ is not bounded below. For any $\varepsilon>0$, there is a real number $d$ which satisfies the condition (*). There is a vector $x_{\varepsilon} \in X$ such that $f\left(x_{\varepsilon}\right)<d$. It is valid that $f\left(x_{\varepsilon}+\varepsilon x\right)>d$ whenever $\|x\|=1, x \in X$. For, if we assume that there is a vector $x \in X,\|x\|=1$ satisfying $f\left(x_{\varepsilon}+\varepsilon x\right) \leqq d$, then we have, by the condition (*), 


$$
\varepsilon>\left\|\left(x_{\varepsilon}+\varepsilon x\right)-x_{\varepsilon}\right\|=\varepsilon\|x\|=\varepsilon,
$$

which is a contradiction. Therefore, we obtain $\inf _{\|x\|=1} f\left(x_{\varepsilon}+\varepsilon x\right) \geqq d>f\left(x_{\varepsilon}\right)$.

Case 2. Consider the case where $f$ is bounded below. Then, there is a real number $d_{0}$ such that $d_{0}=\inf _{x \in X} f(x)$. For every $\varepsilon>0$, there is a real number $d\left(>d_{0}\right)$ which satisfies the condition $(*)$. Then, there exists a vector $x_{\varepsilon} \in X$ such that $f\left(x_{\varepsilon}\right)<d$. By virtue of the argument parallel to that in Case 1, we have $\inf _{|| x \mid i=1} f\left(x_{\varepsilon}+\varepsilon x\right) \geqq d>f\left(x_{\varepsilon}\right)$. Q. E. D.

TheOREM 4.1. Let $X$ be a real Banach space, $H$ a closed convex set in $X$ and $f: X \rightarrow R^{1}$ a uniformly convex function. Under Assumptions 3 and 4 , there exists a unique minimal point for $f$ over $H$ and every minimizing sequence converges (strongly) to the minimal point.

Proof. It turns out that Assumptions 1 and 2 are satisfied, so that all lemmas and propositions in Section 3 still hold. There are two cases.

Case 1. Assume that

$$
d \equiv \inf _{x \in H} f(x)>d_{0} \equiv \inf _{x \in X} f(x) .
$$

If we define the sets $A_{n}$ by

$$
A_{n} \equiv\left\{x \in H \mid f(x) \leqq d+\frac{1}{n}\right\} \quad \text { for } \quad n=1,2, \cdots,
$$

then all $A_{n}$ 's are non-empty, convex sets. It is valid that the decreasing sequence $\left\{A_{n}\right\}$ is a base of Cauchy filter on $H$ since the diameter of the set $A_{n}$ tends to 0 as $n \rightarrow \infty$, by Proposition 3.2. Since the set $H$ is a closed subset of a Banach space $X, H$ is separable and complete, and therefore, the base $\left\{A_{n}\right\}$ of Cauchy filter converges to a unique vector $\bar{x} \in H$. Clearly, $f(\bar{x})=d$. We shall show that the set $A_{\infty} \equiv\{x \in H \mid f(x) \leqq d\}$ consists of a single vector $\bar{x}$. If we suppose that there is a vector $y \in A_{\infty}$ different from $\bar{x}$, then there is a positive number $\varepsilon$ such that $\|y-\bar{x}\| \geqq \varepsilon$. The uniform convexity of $f$ implies that there exists a positive number $\delta(\varepsilon)$ such that $f\left(\frac{x+y}{2}\right) \leqq d-\delta(\varepsilon)<d$. This contradicts to the definition of $d$ since $\frac{x+y}{2} \in H$. Consequently, every minimizing sequence converges to a unique minimum $\bar{x}$.

Case 2. Suppose that $d=d_{0}$ where $d$ and $d_{0}$ are defined in (4.1). Let $A_{n}$ be the set in $X$ defined by (4.2) for $n=1,2, \cdots$. It is valid, on the basis of Proposition 4.1, that the decreasing sequence $\left\{A_{n}\right\}$ is a base of Cauchy filter on $H$ and hence there is a vector $\bar{x} \in X$ to which $\left\{A_{n}\right\}$ converges, such that $f(\bar{x})=d$. It is clear that the uniform convexity of $f$ implies the uniqueness of $\bar{x}$.

Q.E.D.

\section{$\S 5$. An application to the projection theorem in a Hilbert space.}

In this section, we shall show that the preceding arguments combined with nonlinear programming stated in [5] result in the projection theorem in a Hilbert space.

THEOREM 5.1. Let $X$ be a Hilbert space and $A$ a closed convex subset of $X$. If $y$ is a vector in $X$, then there is a unique vector $\bar{x} \in A$ such that

$$
\|y-\bar{x}\|=\min _{x \in A}\|y-x\| .
$$


Moreover, a necessary and sufficient condition for $\bar{x} \in A$ to be a unique minimal vector is that

$$
(y-\bar{x} \mid x-\bar{x}) \leqq 0 \quad \text { for all } \quad x \in A .
$$

Proof. Let $f$ be a real-valued function on $X$ defined by $f(x)=\|y-x\|$. It is easily verified that $f(x)$ is a uniformly convex function in the sense of Definition 3.1 and hence it follows from Theorem 4.1 that there is a unique vector $\bar{x} \in A$ which satisfies (5.1). It is valid that the differential $f_{\bar{x}}(x)$ of $f$ at $\bar{x}$ in the sense of Neustadt is as follows :

$$
f_{\bar{x}}(x)= \begin{cases}\frac{-(y-\bar{x} \mid x)}{\|y-\bar{x}\|} & \text { if } y \neq \bar{x}, \\ \|x\| & \text { if } y=x .\end{cases}
$$

It is then clear, on the basis of Theorem 3.2 in [5], that (5.2) holds if and only if $\bar{x} \in A$ satisfies (5.1).

Q.E.D.

REMARK. If $A$ is a closed subspace of $X$, then (5.2) implies that the vector $y-\bar{x}$ is orthogonal to $A$. Moreover, it may turn out that (5.2) implies the normal equations for the minimization problem.

Acknowledgement. The author is indebted to Prof. Nagata Furukawa of Kyushu University for his useful suggestions.

\section{References}

[1] N. Bourbaki, Espaces Vectoriels Topologiques, Hermann, Paris, 1966. (in Japanese)

[2] E.S. LEvitin and B.T. POLJAK, Convergence of minimizing sequences in conditional extremum problems, Soviet Mathematics, 7 (1966), pp. 764-767.

[3] B. T. POLJAK, Existence theorems and convergence of minimizing sequences in extremum problems with restrictions, Soviet Mathematics, 7 (1966), pp. 72-75.

[4] A. W. Robarts and D.E. Varberg, Convex Functions, Academic Press, 1973.

[5] S. TAGAwA, On a class of nonlinear programming in a Banach space, Bull. Math. Statist., 16 (1974), pp. 95-114.

[6] S. TAGAwA, Generalized convexities of continuous functions and their applications to mathematical programming, Bull. Math. Statist., 16 (1974), pp. 115-125. 